\title{
VELOCIDAD DE PROGRESIÓN DE LA ENFERMEDAD COMO PREDICTOR DE SEVERIDAD EN EL SÍNDROME DE GUILLAIN-BARRÉ
}

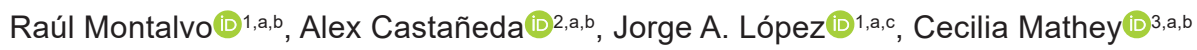 \\ ${ }^{1}$ Facultad de Medicina Humana, Universidad Nacional del Centro del Perú, Huancayo, Perú. \\ ${ }^{2}$ Servicio de Enfermedades Infecciosas y Tropicales, Hospital Lazarte, Trujillo, Perú. \\ ${ }^{3}$ Servicio de Enfermedades Infecciosas y Tropicales, Hospital Daniel A. Carrión, Huancayo, Perú. \\ a Médico/a cirujano/a; ${ }^{b}$ especialista en Enfermedades Infecciosas y Tropicales; ${ }^{c}$ especialista en Radiología y Diagnóstico \\ por imágenes.
}

\section{RESUMEN}

Objetivo: Identificar a la velocidad de progresión de la enfermedad como un predictor de severidad en pacientes con síndrome de Guillain-Barré (SGB). Materiales y métodos: Estudio observacional prospectivo que incluyó pacientes con diagnóstico confirmado de SGB entre mayo y agosto de 2019 en cuatro hospitales del Perú. La velocidad de progresión de la enfermedad (VPE) fue definido como el tiempo transcurrido entre el inicio de los síntomas neurológicos y el pico máximo de la severidad neurológica. Resultados: De 94 casos con SGB, la edad promedio fue 42 años; 73 (77,8\%) pacientes presentaron SGB severo, la estancia hospitalaria promedio fue 18,4 días; 45 (47,8\%) pacientes presentaron cuadro diarreico previo; $63(67,1 \%)$ pacientes presentaron un inicio de debilidad motora en miembros superiores y 31 $(32,9 \%)$ en miembros inferiores; 9 (10,0\%) pacientes presentaron algún tipo de disautonomía; 8 (8,5\%) pacientes debieron usar ventilación mecánica, y $2(2,0 \%)$ pacientes fallecieron. La VPE $\leq 1$ día tiene el $79 \%$ de probabilidad de desarrollar enfermedad severa, la VPE de dos y tres días tienen el $61 \%$ y el 38\% de probabilidad, respectivamente, de progresar a las formas severas. Conclusión: La VPE es un predictor de mal pronóstico cuando es menor de 2 días. La VPE neurológica es un método de evaluación clínica práctica y accesible que debe evaluarse en pacientes con SGB.

Palabras clave: Síndrome de Guillain-Barré; Pronóstico; Índice de Severidad de la Enfermedad; Progresión de la Enfermedad (Fuente: DeCS BIREME).

\section{DISEASE PROGRESSION VELOCITY AS PREDICTOR OF SEVERITY IN GUILLAIN-BARRE SYNDROME}

Citar como: Montalvo R, Castañeda A, López JA, Mathey C. Velocidad de progresión de la enfermedad como predictor de severidad en el síndrome de Guillain-Barré. Rev Peru Med Exp Salud Publica. 2021;38(1):1723. doi: https://doi.org/10.17843/ rpmesp.2021.381.5106.

Correspondencia: Raúl Héctor Montalvo Otivo; Av. Mariscal Castilla 3909, Facultad de Medicina, Universidad Nacional del Centro del Perú, Huancayo, Perú; otivo3@hotmail.com

Recibido: $17 / 01 / 2020$ Aprobado: 04/11/2020 En línea: 28/12/2020

\section{ABSTRACT}

Objective: To identify the velocity of disease progression as a predictor of severity in patients with Guillain-Barre syndrome (GBS). Materials and methods: Prospective observational study of patients with confirmed diagnosis of GBS between May and August 2019 in four hospitals in Peru. The disease progression velocity (DPV) was defined as the time since the onset of neurological symptoms and the maximum peak of neurological severity. Results: Of 94 cases with GBS, the average age was 42 years; 73 (77.8\%) patients presented severe GBS, the average hospital stay was 19 days; 45 (47.8\%) patients had diarrheal symptoms previously, in $63(67.1 \%)$ patients the onset of motor weakness was located in the upper limbs and in 31 (32.9\%) it was located in the lower limbs, 9 (10.0\%) patients presented some type of dysautonomy; admission to mechanical ventilation was needed in $8(8.5 \%)$ patients, and the deceased were $2(2.0 \%)$. The DPV $\leq 1$ day has a $79 \%$ probability of developing severe disease, the two and three day DPV have the probability of $61 \%$ and $38 \%$ respectively of progressing to severe forms. Conclusion: DPV is a predictor of poor prognosis when it is less than 2 days and with a possible requirement for mechanical ventilation. The speed of progression of neurological disease is a practical and accessible clinical evaluation method that should be evaluated in patients with GBS.

Keywords: Guillain-Barre Syndrome; Prognosis; Severity of Illness Index; Disease Progression (Source: MeSH NLM). 


\section{INTRODUCCIÓN}

El síndrome de Guillain-Barré (SGB) es una polineuropatía inflamatoria aguda de etiología autoinmune, de distribución mundial, desencadenada generalmente por agentes infecciosos ${ }^{(1)}$ y caracterizada por la disminución progresiva de la fuerza muscular y de los reflejos osteotendinosos ${ }^{(2)}$. La incidencia global varía entre 0,8 y 3 casos por cada 100000 personas ${ }^{(3,4)}$, la tasa de mortalidad reportada varía entre $3 \%$ y $7 \%$ y la letalidad va de $3 \%$ a $20 \%{ }^{(5,6)}$. En Perú, un estudio epidemiológico de los casos de SGB mostró que la letalidad global fue $3,5 \%$ y esta cifra fue más alta en hombres mayores ${ }^{(7)}$.

Las personas que fallecen por el SGB desarrollan previamente la enfermedad severa caracterizada por la ausencia de la respuesta motora de las cuatro extremidades, insuficiencia respiratoria con requerimiento de ventilación mecánica, fenómenos de disautonomía manifestado por taquicardia, bradicardia, insomnio, ansiedad, palpitaciones, hipotensión ortostática y síncopes ${ }^{(8)}$. Los factores conocidos que condicionan esta severidad son la edad mayor de 60 años, el diagnóstico inadecuado y demora en recibir tratamiento médico ${ }^{(9,10)}$. Sin embargo, existen algunos factores clínicos poco estudiados como la rapidez del deterioro neurológico que podrían estar relacionados con la severidad de la enfermedad.

El objetivo de este estudio fue identificar a la velocidad de progresión de la enfermedad como un predictor de severidad en los pacientes con SGB. Asimismo, de forma exploratoria se describieron las características clínicas y de laboratorio de los pacientes, según sus antecedentes epidemiológicos.

\section{MATERIALES Y MÉTODOS}

\section{Tipo de estudio y población}

Se realizó el estudio observacional prospectivo para enrolar en forma consecutiva a pacientes con diagnóstico de SGB. Se reclutaron participantes de cuatro hospitales peruanos: Hospital Daniel Alcides Carrión de Huancayo (hospital regional especializado en la atención de adultos en enfermedades clínicas y quirúrgicas), Hospital Regional Materno Infantil El Carmen (especializado en la atención de patologías en población pediátrica y materna), Hospital Nacional Ramiro Prialé Prialé (atención general) y Hospital Víctor Lazarte Echegaray - EsSalud de La Libertad (atención general) de mayo a agosto de 2019.

Fueron incluidos pacientes con diagnóstico de SGB, definido mediante los criterios de Brighton ${ }^{(11)}$, que incluyen datos sobre presentación clínica, resultados de laboratorio del líquido cefalorraquídeo (LCR) y hallazgos electrofisiológicos; los casos que no cumplían con estos criterios no fueron incluidos en el estudio, los pacientes eran de cualquier edad y sexo. Las revisiones de los pacientes y de los resultados de laboratorio se realizaron periódicamente hasta el alta

\section{MENSAJES CLAVE}

Motivación para realizar el estudio: El síndrome de GuillainBarré puede ocasionar complicaciones severas, por lo que es relevante identificar los predictores de severidad; el más importante es la velocidad de progresión de la enfermedad (VPE), definido como el tiempo desde el inicio de la debilidad hasta el pico máximo de la severidad neurológica.

Principales hallazgos: Pacientes con una VPE $\leq 1$ día tiene $80 \%$ de probabilidad de desarrollar enfermedad severa, aquellos con una VPE $\leq 2$ días tiene la probabilidad de $61 \%$ de avanzar a las formas severas..

Implicancias: La VPE es un predictor de mal pronóstico y con posible requerimiento de ventilación mecánica por parte de los pacientes, que debe incluirse en la anamnesis.

hospitalaria. Los datos recopilados se obtuvieron mediante una ficha de extracción de datos previamente establecida que incluyeron datos demográficos, antecedentes de enfermedades, examen físico neurológico, resultados de laboratorio, estudio de electromiografía y estado de alta hospitalaria. Se calculó el puntaje de discapacidad de Hughes al ingreso y al alta para definir la severidad de la enfermedad ${ }^{(12)}$. El seguimiento de los pacientes se realizó hasta el alta hospitalaria o hasta su fallecimiento.

\section{Variables}

La variable de interés fue la velocidad de progresión de la enfermedad (VPE), estimada por la diferencia en días del tiempo transcurrido entre el inicio de los síntomas y el pico máximo de la severidad neurológica, para esto se consideraron como hitos el día en que ingresó a ventilación mecánica; el día en que se detuvo la progresión de la enfermedad o el día de fallecimiento del paciente antes de ingresar a ventilación mecánica.

Otras variables de interés fueron edad, sexo, comorbilidad, gestación, resultados de laboratorio de hematología, resultado del LCR, características clínicas de ingreso. Los datos se obtuvieron de la entrevista directa, del examen físico realizado por los investigadores a los pacientes que acudían por presentar debilidad motora progresiva y disminución de los reflejos osteotendinosos, y de los resultados de laboratorio obtenido de la revisión de las historias clínicas de los pacientes con SGB.

\section{Análisis estadístico}

Para el proceso de doble entrada de datos se utilizó Microsoft Excel para Windows, y para el análisis se usó el programa estadístico Stata versión 12 (STATA Corp, College Station, TX, EUA). El análisis estadístico se realizó con una descripción 
de las características demográficas y clínicas, tabulándolas de acuerdo con la escala de Hughes. Las variables categóricas se compararon utilizando la prueba Chi cuadrado o la prueba exacta de Fisher según corresponda, mientras que las variables numéricas se compararon mediante la prueba $t$ de Student. Asimismo, el promedio de la VPE, el promedio de los resultados del hemograma, promedio de los resultados del LCR y de la gasometría se calcularon para cada grupo según la severidad del SGB.

Para el análisis multivariado, se consideró como variables independientes: edad en años, sexo, características clínicas y demográficas, antecedentes de enfermedad, resultados de análisis hematológico, bioquímico, gases arteriales y resultados del LCR. Las variables que presentaron asociación significativa en el análisis bivariado e incluidas en el modelo fueron presión de $\mathrm{CO} 2$, arreflexia bicipital, velocidad de progresión de la enfermedad, monocitosis en el hemograma, edad y sexo los cuales fueron categorizados de acuerdo a estudios previos y a los valores referenciados ${ }^{(9-10)}$. Se realizó el análisis de múltiple variable considerando como variable dependiente la escala de Hughes $>3$ como punto de corte que determina la severidad del SGB. Se calcularon los riesgos relativos (RR) con intervalos de confianza al 95\% (IC 95\%) y se consideró un valor de $\mathrm{p}<0,05$ para indicar la significancia estadística.

Para evaluar la probabilidad de desarrollar un cuadro severo en un SGB según la VPE, se estimaron los puntos corte de la VPE; utilizando curvas ROC (por sus siglas en inglés), se determinó la probabilidad de desarrollar una enfermedad severa según cada punto de corte en días de la VPE y se obtuvo el mejor punto de corte de la VPE. Se utilizó el comando roctab del programa estadístico Stata versión 12 (StataCorp, College Station, Texas, EUA), con el objetivo de evaluar la capacidad de predecir la severidad de SGB.

\section{Aspectos éticos}

Los comités de ética en investigación del Hospital Daniel Alcides Carrión (Exp. No: 379-19) y del Hospital Nacional Ramiro Prialé (Exp. $N^{\circ}:$ 143-19) aprobaron el desarrollo del estudio. Asimismo, todos los hospitales autorizaron el uso de la información de los pacientes. Finalmente, la investigación se realizó cumpliendo las normas éticas internacionalmente exigidas, respetándose la confidencialidad de los pacientes.

\section{RESULTADOS}

De 121 pacientes que ingresaron al estudio, 27 (22\%) se perdieron durante en el seguimiento debido a lo siguiente: altas voluntarias en $9(0,7 \%)$ pacientes; $11(0,9 \%)$ fueron referidos a otro centro hospitalario público o privado; y $7(0,6 \%)$ fueron diagnosticados de otra enfermedad durante el seguimien- to hospitalario, como encefalitis, polineuropatía infecciosa o enfermedad cerebrovascular. Se evaluaron finalmente 94 casos con SGB, la edad promedio fue 42 años (rango 8-73); $77,8 \%$ presentaron SGB severo (escala de Hughes $>3$ ); 64 (68\%) fueron varones; la estancia hospitalaria promedio fue 19 días (rango 1-129). El promedio del tiempo de enfermedad al ingreso fue 4 días; 12 (12,8\%) pacientes presentaron alguna comorbilidad; $3 \%$ recibieron algún tipo de vacunación el último mes antes de su ingreso; el promedio del índice de masa corporal (IMC) fue 26,4 (Tabla 1).

Los síntomas infecciosos previo a la debilidad muscular estuvieron presentes en 71 (81\%) pacientes con SGB, de los cuales $45(47,8 \%)$ presentaron cuadro diarreico y $22(23,4 \%)$ mostraron un síndrome gripal (Tabla 1). La duración de estos síntomas fue 3 días en promedio. El tiempo promedio trascurrido entre el inicio de estos síntomas y el inicio de la debilidad motora fue 5 días (rango 1-21).

El lugar de inicio de la debilidad motora en los miembros superiores se evidenció en $63(67,1 \%)$ pacientes y 31 $(32,9 \%)$ pacientes iniciaron con debilidad en los miembros inferiores (Tabla 1). La distribución por el lugar de inicio de la debilidad no fue significativamente diferente en comparación con la severidad del SGB ( $\mathrm{p}=0,121)$.

El tratamiento farmacológico administrado fue inmunoglobulina en $86(91,0 \%)$ pacientes y plasmaféresis en $6(7,0 \%)$ pacientes (Tabla 1). El promedio de la escala de Hughes al egreso hospitalario fue 2 (rango 0-5). Con relación a las complicaciones, $9(10,0 \%)$ pacientes presentaron algún tipo de disautonomía. El ingreso a ventilación mecánica fue necesario para $8(8,5 \%)$ pacientes.

El análisis del LCR no evidenció resultados asociados a la severidad del SGB (Tabla 2); los valores de la presión de dióxido de carbono $\left(\mathrm{PCO}_{2}\right)$ evidenciados en el análisis de gases arteriales y el recuento de monocitos en el hemograma fueron más elevados en SGB severo ( $\mathrm{p}=0,001$ y $\mathrm{p}=0,023$, respectivamente).

En el análisis multivariado, la VPE por más de 3 días se comportó como un factor protector para no desarrollar enfermedad severa $(R R=0,27$; IC95\%: 0,07 - 0,95; valor de $\mathrm{p}=$ 0,041) (Tabla 3). Asimismo, se evidencia que a menor valor de la VPE se incrementa la probabilidad de que la enfermedad se agrave (Tabla 4 ).

\section{DISCUSIÓN}

Con el presente estudio se identificó que el factor más importante que condiciona el curso de la enfermedad severa en el SGB es la VPE. La escala que se usó para determinar el grado de severidad fue la escala de Hughes ${ }^{(12)}$. Este estudio mostró que $78 \%$ de los pacientes con SGB ingresaron en silla de ruedas 
Tabla 1. Características generales de los pacientes con síndrome de Guillain-Barré.

\begin{tabular}{|c|c|c|c|c|c|}
\hline \multirow[b]{2}{*}{ Características generales } & \multirow[b]{2}{*}{$\mathrm{n}=94$} & \multirow[b]{2}{*}{$\%$} & \multicolumn{3}{|c|}{ Distribución de los pacientes según escala de Hughes } \\
\hline & & & $\begin{array}{l}\text { Hughes } \leq 3 \\
\text { n }(\%)\end{array}$ & $\begin{array}{c}\text { Hughes }>3 \\
\text { n }(\%)\end{array}$ & Valor de $\mathrm{p}$ \\
\hline Edad: promedio en años (rango) & 42 & $(8-73)$ & $41(10-81)$ & $42(8-73)$ & 0,972 \\
\hline$<40$ & 36 & 38,0 & $11(52,4)$ & $25(34,3)$ & 0,033 \\
\hline $40-59$ & 45 & 48,0 & $5(23,8)$ & $40(54,8)$ & \\
\hline$>59$ & 13 & 14,0 & $5(23,8)$ & $8(10,9)$ & \\
\hline Sexo: masculino & 64 & 68,0 & $9(42,9)$ & $55(75,4)$ & 0,008 \\
\hline Estancia hospitalaria: promedio (rango) & 18,4 & $(1-129)$ & $10,3(1-24)$ & $22,5(1-129)$ & 0,110 \\
\hline Tiempo de enfermedad: promedio (rango) ${ }^{\mathrm{a}}$ & 4 & $(1-8)$ & $4(1-8)$ & $3(0,5-7)$ & 0,077 \\
\hline Comorbilidades & 12 & 12,8 & $3(10,4)$ & $9(12,5)$ & 0,527 \\
\hline Vacunación anterior & 3 & 3,3 & $1(4,7)$ & $2(2,7)$ & 0,091 \\
\hline IMC: promedio (rango) & 26,4 & $(18-45)$ & $25(18-31)$ & $26(19-45)$ & 0,242 \\
\hline$<18,5$ & 3 & 3,2 & $3(14,3)$ & $0(0,0)$ & 0,276 \\
\hline $18,5-24,9$ & 28 & 29,8 & $6(28,6)$ & $22(30,1)$ & \\
\hline $25-29,9$ & 47 & 50,0 & $10(50,0)$ & $37(50,7)$ & \\
\hline$\geq 30$ & 16 & 17,0 & $2(7,1)$ & $14(19,2)$ & \\
\hline \multicolumn{6}{|l|}{ Síntomas previos ${ }^{\mathrm{b}}$} \\
\hline Diarrea & 45 & 47,8 & $12(57)$ & $33(47)$ & 0,303 \\
\hline Síndrome gripal & 22 & 23,4 & $3(14)$ & $19(26)$ & \\
\hline Fiebre & 4 & 4,3 & $2(10)$ & $2(3)$ & \\
\hline Ninguno & 18 & 19,2 & $4(19)$ & $14(19)$ & \\
\hline Duración de síntomas: promedio (rango) & 3,3 & $(1-10)$ & $4,6(1-10)$ & $3(1-7)$ & 0,062 \\
\hline $\begin{array}{l}\text { Días entre el inicio de síntomas y debilidad: promedio } \\
\text { (rango) }\end{array}$ & 5 & $(1-21)$ & $5,7(1-15)$ & $4,2(0-21)$ & 0,237 \\
\hline \multicolumn{6}{|l|}{ Tipo de arreflexia } \\
\hline Arreflexia rotuliana & 16 & 17,1 & $8(38,1)$ & $8(10,9)$ & 0,003 \\
\hline Arreflexia bicipital & 14 & 14,9 & $4(19,1)$ & $10(13,7)$ & \\
\hline Arreflexia rotuliana y bicipital & 37 & 39,4 & $4(19,1)$ & $33(45,3)$ & \\
\hline Otros tipos de arreflexia & 27 & 28,6 & $5(23,7)$ & $22(30,1)$ & \\
\hline \multicolumn{6}{|l|}{ Tipo de polineuropatía } \\
\hline Compromiso motor & 59 & 62,7 & $17(94,4)$ & $42(87,5)$ & 0,660 \\
\hline Compromiso motor y sensitivo & 7 & 7,5 & $1(5,6)$ & $6(12,5)$ & \\
\hline $\begin{array}{l}\text { Velocidad de Progresión de enfermedad (VPE): pro- } \\
\text { medio (rango) }\end{array}$ & 3,6 & $(1-9)$ & $5(1-9)$ & $3(0,5-7)$ & 0,006 \\
\hline \multicolumn{6}{|l|}{ Lugar de inicio } \\
\hline Miembros inferiores & 31 & 32,9 & $10(47,6)$ & $21(28,8)$ & 0,121 \\
\hline Miembros superiores & 63 & 67,1 & $11(52,4)$ & $52(71,2)$ & \\
\hline Escala de Hughes al ingreso: promedio (rango) & 3 & $(1-5)$ & $1,7(1-2)$ & $3,4(2-5)$ & $<0.01$ \\
\hline \multicolumn{6}{|l|}{ Tratamiento } \\
\hline Inmunoglobulina & 86 & 91,0 & $19(90,5)$ & $67(91,8)$ & 0,024 \\
\hline Plasmaféresis & 6 & 7,0 & 0 & $6(8,2)$ & \\
\hline Ninguno & 2 & 2,0 & $2(9,5)$ & 0 & \\
\hline \multicolumn{6}{|l|}{ Egreso hospitalario } \\
\hline Fallecido & 2 & 2,0 & 0 & $2(2,7)$ & 0,58 \\
\hline Escala de Hudge al egreso: promedio (rango) & 2 & $(0-5)$ & $1,4(1-2)$ & $2,5(1-5)$ & 0,003 \\
\hline \multicolumn{6}{|l|}{ Complicaciones } \\
\hline Disautonomía & 9 & 10,0 & 0 & $9(12,3)$ & 0,034 \\
\hline Ventilación mecánica $^{\mathrm{d}}$ & 8 & 8,5 & 0 & $8(10,9)$ & 0,192 \\
\hline
\end{tabular}

${ }^{a}$ Días entre el inicio de debilidad y el ingreso al hospital. ${ }^{\mathrm{b}}$ Cinco pacientes presentaron síntomas inespecíficos como malestar general, hiporexia, cefalea, dolor abdominal. ${ }^{\mathrm{c}}$ Tiempo en días desde el inicio de la debilidad y el pico máximo de la severidad neurológica (VPE). ${ }^{\mathrm{d}}$ Dos pacientes continuaron en ventilador hasta el cierre del periodo de estudio. IMC: índice de masa corporal. 
Tabla 2. Comparación de los parámetros de laboratorio al ingreso hospitalario de los pacientes con síndrome de Guillain-Barré.

\begin{tabular}{|c|c|c|c|c|}
\hline Parámetros & Promedio (rango) & $\begin{array}{c}\text { Hughes } \leq 3 \\
\text { promedio (rango) }\end{array}$ & $\begin{array}{c}\text { Hughes }>3 \\
\text { promedio (rango) }\end{array}$ & Valor de $\mathrm{p}^{\mathrm{b}}$ \\
\hline \multicolumn{5}{|l|}{$\mathrm{LCR}^{\mathrm{a}}$} \\
\hline Leucocitos $\left(\mathrm{mm}^{3}\right)$ & $7,9(0-47)$ & $6,1(0-43)$ & $8,7(0-47)$ & 0,572 \\
\hline Polimorfonucleares (\%) & $13,4(0-30)$ & $3,3(0-30)$ & $5,1(15-30)$ & 0,554 \\
\hline Mononucleares (\%) & $89,4(0-100)$ & $76,8(0-100)$ & $95,1(70-100)$ & 0,051 \\
\hline Adenosinadesaminasa (U/L) & $8(4-11,7)$ & $7,4(4-10)$ & $7,8(4-11.7)$ & 0,744 \\
\hline Proteínas (mg/dL) & $52(10-193)$ & $43,7(10-119)$ & $56(10-193)$ & 0,383 \\
\hline Glucosa (mg/dL) & $54,5(41-74)$ & $54,6(46-74)$ & $54,5(41-72)$ & 0,971 \\
\hline \multicolumn{5}{|l|}{ Hemograma al ingreso } \\
\hline Leucocitos $\left(\mathrm{mm}^{3}\right)$ & $8124,9(3060-17$ 100) & $7089(5030-10600)$ & $8320(3060-17$ 100) & 0,143 \\
\hline Neutrófilos $\left(\mathrm{mm}^{3}\right)$ & $5381,8(440-13950)$ & $4957(2820-7150)$ & $5311(440-13950)$ & 0,714 \\
\hline Eosinófilos $\left(\mathrm{mm}^{3}\right)$ & $128,6(0-910)$ & $106(0-248)$ & $134(0-910)$ & 0,551 \\
\hline Basófilos $\left(\mathrm{mm}^{3}\right)$ & $67,6(0-910)$ & $53,3(0-560)$ & $73,8(0-910)$ & 0,612 \\
\hline Monocitos $\left(\mathrm{mm}^{3}\right)$ & $463,8(0-1539)$ & $364(82-636)$ & $484(40-850)$ & 0,023 \\
\hline Linfocitos $\left(\mathrm{mm}^{3}\right)$ & 2097,3 (154-3920) & $2049(390-3286)$ & $2135(154-3920)$ & 0,753 \\
\hline Abastonados $\left(\mathrm{mm}^{3}\right)$ & $53,2(0-1368)$ & $18(14-150)$ & $63,3(0-1368)$ & 0,413 \\
\hline Plaquetas $\left(\mathrm{mm}^{3}\right)$ & $336682,5(170000-501000)$ & $299500(192000-395000)$ & $289425(170000-501000)$ & 0,662 \\
\hline \multicolumn{5}{|l|}{ Gasometría y bioquímica } \\
\hline $\mathrm{PO}_{2}(\mathrm{mmHg})$ & $69,2(51-85.9)$ & $73,2(72-74,4)$ & $69,2(51-85,9)$ & 0,524 \\
\hline $\mathrm{PCO}_{2}(\mathrm{mmHg})$ & $30,3(20-39,3)$ & $38,9(25,7-44)$ & $30,8(21,7-39,3)$ & 0,001 \\
\hline $\mathrm{FiO}_{2}(\%)$ & $22,9(21-50)$ & $21(21-21)$ & $23,1(24-50)$ & 0,663 \\
\hline Sodio $(\mathrm{mmol} / \mathrm{L})$ & $139,3(132-148)$ & $135(132-138)$ & $139(133-148$ & 0,131 \\
\hline Calcio (mmol/L) & $1,2(0,9-1,85)$ & $1,2(1,1-1,19)$ & $1,2(0,9-1,85)$ & 0,812 \\
\hline Deshidrogenasa láctica (U/L) & $370,4(42,4-900)$ & $115(42,4-188)$ & $370(351-900)$ & 0,213 \\
\hline Transaminasa (TGO U/L) & $45,2(14-170)$ & $26,7(25,2-27,8)$ & $50,3(14-170)$ & 0,390 \\
\hline Transaminasa (TGP U/L) & $65,1(16-313)$ & $27,3(24-30,5)$ & $72,2(16-313)$ & 0,511 \\
\hline Urea (mg/dL) & 32,3 & $26,1(19,3-31)$ & $32,2(20,6-84,6)$ & 0,183 \\
\hline Creatinina (mg/dL) & 0,9 & $0,8(0,59-0,96)$ & $0,9(0,14-1,29$ & 0,324 \\
\hline Proteínas totales $(\mathrm{g} / \mathrm{dL})$ & 7,4 & $7,2(6,3-7,9)$ & $7,6(5,9-10,1)$ & 0,532 \\
\hline Glucosa (mg/dL) & 94,8 & $96,4(81-123)$ & $94,2(71-139)$ & 0,764 \\
\hline $\operatorname{PCR}(\mathrm{mg} / \mathrm{dL})$ & $24,7(0,23-96)$ & $14,4(0,23-62)$ & $34,7(3-96)$ & 0,481 \\
\hline
\end{tabular}

a Promedio de los resultados del LCR tomado al día 7 de enfermedad. b Se utilizó la prueba t de Student para calcular el valor de p.

LCR: líquido cefalorraquídeo; PCR: proteína C reactiva; TGO: transaminasa glutámico-oxalacética; TGP: alanina aminotransferasa; FiO 2 : fracción inspirada de oxígeno; $\mathrm{PO}_{2}$ : presión parcial de oxígeno medido en $\mathrm{mmHg}$.

o con permanencia en cama (Hughes $>3$ ), este porcentaje de severidad fue similar a los datos obtenidos por Yosria et al. ${ }^{(13)}$ y Parmar et al. ${ }^{(14)}$ quienes reportaron que la mayoría de los pacientes atendidos con SGB tuvieron el grado $\geq$ IV, con $75 \%$ y 83,7\% respectivamente. Estos autores explican que la razón de la alta frecuencia de severidad de la enfermedad fue por el retraso en la administración del tratamiento. Este estudio confirmó que la velocidad de progresión de la debilidad está asociada a la severidad, mostrando que los pacientes con VPE menor o igual a un día tienen cerca de $80 \%$ de probabilidad de desarrollar la enfermedad severa.
El promedio de la VPE fue tres días en los pacientes con SGB severo, similar a un estudio ${ }^{(15)}$, que además encontró concentraciones elevadas de anticuerpos contra los gangliósidos GM1, GD1a, GalNac-GD1a y Gd1b en los axones nerviosos periféricos, estos anticuerpos pueden ser inducidos por agentes infecciosos como Campylobacter jejuni ${ }^{(16)}$, que producen daño en el segmento de la vaina de mielina y a la raíz nerviosa proximal ${ }^{(6)}$.

Varios estudios coinciden que casos severos de SGB puede ocurrir a cualquier edad e indistintamente del sexo ${ }^{(15)}$. A pesar de que otros trabajos señalan que, por lo general, el SGB es más 
Tabla 3. Predictores de severidad en pacientes con síndrome de Guillain-Barré.

\begin{tabular}{|c|c|c|c|c|}
\hline \multirow{2}{*}{ Variable } & Modelo crudo & \multirow{2}{*}{ Valor de p } & Modelo multivariado $^{a}$ & \multirow{2}{*}{ Valor de $\mathrm{p}$} \\
\hline & RR (IC 95\%) & & RR (IC 95\%) & \\
\hline \multicolumn{5}{|c|}{ Presión de $\mathrm{CO}_{2}(\mathrm{mmHg})^{*}$} \\
\hline$<32$ & Referencia & & Referencia & \\
\hline$\geq 32$ & $0,61(0,31$ a 0,96$)$ & 0,011 & $0,58(0,34$ a 1,00$)$ & 0,050 \\
\hline \multicolumn{5}{|l|}{ Reflejo bicipital } \\
\hline Presente & Referencia & & Referencia & \\
\hline Ausente & $4,93(1,01$ a 29,7$)$ & 0,037 & $4,69(0,78$ a 28,0$)$ & 0,091 \\
\hline \multicolumn{5}{|c|}{ Velocidad de progresión (días) } \\
\hline$\leq 3$ & Referencia & & Referencia & \\
\hline$>3$ & $0,39(0,06$ a 0,91$)$ & 0,002 & $0,27(0,07$ a 0,95$)$ & 0,041 \\
\hline \multicolumn{5}{|c|}{ Monocitos (/mL) } \\
\hline$\leq 500$ & Referencia & & Referencia & \\
\hline$>500$ & $1,17(1,01$ a 1,33$)$ & 0,023 & $1,01(0,98$ a 1,01$)$ & 0,082 \\
\hline \multicolumn{5}{|l|}{ Sexo } \\
\hline Masculino & Referencia & & Referencia & \\
\hline Femenino & $0,08(-16,91$ a 5,01$)$ & 0,084 & $0,03(-17,10$ a 5,32$)$ & 0,303 \\
\hline \multicolumn{5}{|l|}{ Edad (años) } \\
\hline$\geq 60$ & Referencia & & Referencia & \\
\hline$<60$ & $0,98(-2,91$ a 2,02$)$ & 0,972 & $0,97(-3,28$ a 1,33$)$ & 0,407 \\
\hline
\end{tabular}

ajustado por comorbilidad, tratamiento y lugar de inicio de la debilidad. RR: riesgo relativo; IC 95\%: intervalo de confianza al 95\%.

frecuente en hombres y con un promedio de edad que varía de 32 a 44 años ${ }^{(17-18)}$, hallazgos similares se han evidenciado en el presente estudio, donde $68 \%$ de los afectados fueron varones y el promedio de edad fue 42 años, sin embargo, estas dos características no estuvieron asociados a la severidad de la enfermedad.

De acuerdo con el estudio de Mao et al. ${ }^{(19)}$ la tasa de letalidad por SGB es 6\%, según los hallazgos de este estudio la tasa de letalidad fue $2 \%$, mucho menor que lo encontrado en otros estudios, esta baja tasa podría deberse a la forma de presentación típica. Las causas de fallecimiento estuvieron relacionados con la demora del ingreso al ventilador mecánico o con la exposición prolongada a este. En el presente estudio, fallecieron 2 pacientes,

Tabla 4. Probabilidad de desarrollar enfermedad severa en el síndrome de Guillain-Barré según velocidad de progresión de enfermedad.

\begin{tabular}{lcc}
\hline $\begin{array}{l}\text { Velocidad de } \\
\text { progresión de } \\
\text { enfermedad (días) }\end{array}$ & $\begin{array}{c}\text { Probabilidad } \\
\text { de desarrollar } \\
\text { enfermedad severa }\end{array}$ & IC 95\% \\
\hline$\leq 1$ & 0,79 & $0,64-0,89$ \\
2 & 0,61 & $0,45-0,74$ \\
3 & 0,38 & $0,25-0,53$ \\
4 & 0,20 & $0,10-0,33$ \\
5 & 0,06 & $0,02-0,17$ \\
6 & 0,04 & $0,01-0,14$ \\
\hline
\end{tabular}

IC 95\%: intervalos de confianza al 95\% uno de ellos ingresó en mal estado general y falleció el primer día de internamiento por la demora en su ingreso a ventilador mecánico y el segundo por complicaciones infecciosas por Acinetobacter baumanni panresistente debido al uso prolongado de ventilador mecánico el día 112 de hospitalización.

La complicación más frecuente fue la presencia de disautonomía que se evidenció en 19\% de los pacientes con SGB severo. Existen otras características clínicas y laboratoriales en los pacientes con SGB severo como la arreflexia bicipital, valores de $\mathrm{PCO}^{2}>30$ y monocitos $>490$, pero que no fueron significativamente más altos en el grupo de pacientes con SGB severo cuando se realizó el análisis multivariado.

Los hallazgos del estudio de LCR evidencian linfocitos normales o ligeramente elevados $<50$ células $/ \mathrm{mm}^{3}$ y que los niveles de proteínas se elevan a partir de la primera semana de enfermedad, esto está presente en más del $90 \%$ de los afectados por SGB ${ }^{(20)}$. El presente estudio muestra los resultados del análisis de LCR a la primera semana de ingreso; el promedio de los valores de proteína en el LCR fue $52 \mathrm{mg} / \mathrm{dL}$ y la disociación albuminocitológica se vio en el $90 \%$ de los casos. La elevación de proteínas en el LCR se debe al aumento de los depósitos de anticuerpos, complementos y productos de mielina al descomponerse, mientras que el nivel de IgG y anticuerpos antigangliósidos están relacionados con el pronóstico de la enfermedad ${ }^{(21)}$.

La velocidad de progresión de la enfermedad neurológica es un método de evaluación práctica y accesible que debe ser considerada por los médicos e incluida en las escalas de predicción de severidad de SGB. En este estudio, se observó que 
cuando el valor es igual o menor a un día, la probabilidad de que el paciente desarrolle la forma severa es $80 \%$ aproximadamente y esta probabilidad se reduce a $60 \%$ si es dos días, comportándose como un predictor de mal pronóstico cuando la VPE es menor de dos días y con posible requerimiento de ventilación mecánica.

La fortaleza de estudio radica en la naturaleza de la recolección de datos de manera longitudinal para evaluar la velocidad de progresión de la enfermedad como predictor de severidad de SGB. Sin embargo, este estudio tiene algunas limitaciones. Primero, las pérdidas de pacientes durante los meses de seguimiento que representó más de $20 \%$; por lo que es necesario averiguar que sucedió con esos pacientes perdidos para evaluar la naturaleza del resultado final, fallecido o recuperado. Segundo, hay algunos datos que no fueron explorados a profundidad, como el estudio de electromiografía, por la falta de equipos que realicen dicho estudio en algunos hospitales o por la elevada demanda de este equipo en el periodo del brote de SGB. Finalmente, si bien presentamos una fuerte correlación entre
VPE y severidad de SGB, nuestros hallazgos deberían verificarse con un estudio prospectivo con mayor número de pacientes que incluyan análisis serológicos de marcadores inflamatorios.

Los resultados del presente estudio apoyan la hipótesis de que, en los pacientes con SGB, debe evaluarse la VPE neurológica y que la duración menor de dos días se encontró asociada a la alta probabilidad de presentar enfermedad severa, reflejando así el impacto de VPE en la gravedad y su posible utilidad como marcador pronóstico.

Contribuciones de los autores: RM, AC, JL y CM participaron en la concepción del artículo, la recolección de datos, el análisis y la interpretación de datos, en la redacción y revisión crítica del manuscrito, y en la aprobación de la versión final. Todos los autores son responsables del contenido del artículo.

Financiamiento: Autofinanciado.

Conflictos de interés: No existen conflictos de interés para la publicación del artículo.

\section{REFERENCIAS BIBLIOGRÁFICAS}

1. Montalvo R, García Y, Navincopa M, Ticona E, Chávez G, Moore DA. Síndrome de Guillain Barré asociado a Brucelosis. Rev Peru Med Exp Salud Publica. 2010;27(2):292-295. doi: 10.1590/s1726-46342010000200020.

2. Willison HJ, Jacobs BC, van Doorn PA. Guillain-Barré syndrome. Lancet. 2016;388(10045):717-727. [citado 27 diciembre 2019]. doi: 10.1016/S01406736(16)00339-1.

3. Sejvar JJ, Baughman AL, Wise M, Morgan OW. Population incidence of Guillain-Barré syndrome: A systematic review and meta-analysis. Neuroepidemiology. 2011;36:123-133. doi: https://doi.org/10.1159/000324710.

4. McGrogan A, Madle GC, Seaman HE, de Vries CS. The epidemiology of Guillain-Barré syndrome worldwide: a systematic literature review. Neuroepidemiology. 2009;32(2):150-163. doi: https://doi. org/10.1159/000184748.

5. Salinas JL, Major CG, Pastula DM, Dirlikov E, Styczynski A, Luciano CA et al. Incidence and clinical characteristics of Guillain-Barré syndrome before the introduction of Zika virus in Puerto Rico. J Neurol Sci. 2017;377:102106. doi: 10.1016/j.jns.2017.04.006.

6. Hughes RA, Swan AV, Raphaël JC, Annane D, van Koningsveld R, van Doorn PA. Immunotherapy for Guillain-Barré syndrome: a systematic review. Brain. 2007;130(Pt 9):2245-57. doi: 10.1093/brain/awm004.

7. Munayco CV, Soto MG, Reyes MF, Arica JA, Napanga O. Epidemiología del síndrome de Guillain-Barré en el Perú. Rev Peru Med Exp Salud Publica. 2019;36(1):10-6. doi: 10.17843/rpmesp.2019.361.3729.

8. De Boisanger L. Outcomes for patients with Guillain-Barré syndrome requiring mechanical ventilation: a literature review. Ir J Med Sci. 2016;185:11-15. doi: 10.1007/s11845-015-1365-7.

9. Arsenijević M, Berisavac I, Mladenović B. Rate of progression of Guillain-Barré syndrome is not associated with the short-term outcome of the disease. Ir J Med Sci. 2020;10.1007/s11845-020-02310-7. doi: 10.1007/ s11845-020-02310-7.

10. López-Hernández JC, Colunga-Lozano LE, Garcia-Trejo S, Gomez-Figueroa E, Delgado-Garcia G, Bazán-Rodríguez L. Electrophysiological subtypes and associated prognosis factors of Mexican adults diagnosed with Guillain-Barré syndrome, a single center experience. J Clin Neurosci. 2020:S0967-5868(20)30394-5. doi: 10.1016/j.jocn.2020.04.059.

11. Sejvar JJ, Kohl KS, Gidudu J, Amato A, Bakshi N, Baxter R, et al. Guillain-Barré syndrome and Fisher syndrome: case definitions and guidelines for collection, analysis, and presentation of immunization safety data. Vac- cine. 2011;29(3):599-612. doi: 10.1016/j.vaccine.2010.06.003.

12. Hughes RA, Newsom-Davis JM, Perkin GD, Pierce JM. Controlled trial prednisolone in acute polyneuropathy. Lancet. 1978;2(8093):750-753. doi: 10.1016/s0140-6736(78)92644-2.

13. Altaweel YA, Abdelaziz S, Fathy HA, AbdelBadea S. Correlative study between C-reactive protein, clinical severity, and nerve conduction studies in Guillain-Barrè syndrome. Egypt J Neurol Psychiatr Neurosurg. 2018;54(1):4. doi: 10.1186/s41983-018-0006-2.

14. Parmar LD, Doshi V, Singh SK. Nerve conduction studies in Guillian Barré syndrome. Int J Neurol. 2013;16(1):1-14.

15. Van den Berg, Storm F, Garssen M, Blomkwist-Markens, Jacobs B. Clinical outcome of Guillain-Barré syndrome after prolonged mechanical ventilation. J Neurol Neurosurg Psychiatry 2018;0:1-6. doi: 10.1136/jnnp-2018-317968.

16. Kokubun N, Nishibayashi M, Uncini A. Conduction block in acute motor axonal neuropathy. Brain. 2010;133:2897. doi: 10.1093/brain/awq260.

17. Van den Berg B, Walgaard C, Drenthen J, Fokke C, Jacobs BC, van Doorn PA. Guillain-Barré syndrome: pathogenesis, diagnosis, treatmentandprognosis. Nat Rev Neuro. 2014;10:469-82. doi: 10.1038/ nrneurol.2014.121.

18. Ritter C, Bobylev I, Lehmann HC. Chronic inflammatory demyelinating polyneuropathy (CIDP): Change of serum IgG dimer levels during treatment with intravenous immunoglobulins. J Neuroinflammation 2015;12:148. doi: 10.1186/s12974-015-0361-1.

19. Mao Z, Hu X. Clinical characteristics and outcomes of patients with Guillain- Barre and acquired CNS demyelinating overlap syndrome: a cohort study based on a literature review. Neurol Res. 2014;36(12):1106-13. doi: 10.1179/1743132814Y.0000000400.

20. Islam B, Islam Z, Rahman S, Endtz HP, Vos MC, van der Jagt M, et al. Small volume plasma exchange for Guillain-Barré syndrome in resourcelimited settings: a phase II safety and feasibility study. BMJ Open 2018;8:e22862. doi: 10.1136/bmjopen-2018-022862.

21. ApazaEL. Características clínicas y electrofisiológicas del síndrome de Guillain Barré en el Instituto Nacional de Ciencias Neurológicas, 2008-2012. Tesis para obtener el título de especialista en Neurología. Lima: Universidad Nacional Mayor de San Marcos; 2014. Disponible en: https://cybertesis.unmsm.edu.pe/ bitstream/handle/20.500.12672/12920/Apaza_Nina_Littman_2014.pdf?sequence $=1$ \&isAllowed $=\mathrm{y}$. 